\title{
Stochastic Dominance and Comparative Risk Aversion
}

\author{
TAPAN BISWAS \\ University of Hull*
}

\section{Introduction}

The quest for criteria to rank risky alternatives led to the development of the literature on stochastic dominance. Given a set of utility functions $U$ and two uncertain prospects $g^{1}($.) and $g^{2}($.$) , the rule of stochastic dominance provides the condition under which an individual$ $i$ will always prefer $g^{1}\left(\right.$.) to $g^{2}($.) so long as his utility function is a member of $U$. The rule of stochastic dominance depends on the choice of the set $U$. Quirk and Saposnik (1962) started with the choice of $U$ as the set of increasing utility functions. Hadar and Russell (1969) considered the case of $U$ consisting of all concave (risk-averse) utility functions. Whitmore (1970) further restricted the choice of utility functions by considering $U$ as the set of declining risk-averse utility functions. Meyer (1977) introduced the concept of second degree stochastic dominance with respect to a function which generalizes the work of Russell and Hadar. For further extension of Meyer's work, the reader is referred to Bradley and Lehman (1985).

Here, we look at the rules of stochastic dominance from a different point of view. Consider $g()=.\left\{g^{i}().\right\}$ as the set of available lotteries or uncertain prospects and $n$ individuals having utility functions $u^{i}(),. i=1,2 \ldots n$. Suppose the pair of lotteries $\left(g^{k}(),. g^{h}().\right)$ is such that the $i$-th individual prefers $g^{k}\left(\right.$.) to $g^{h}($.$) . The intersection of the set of all such ordered pairs$ for all the individuals is called the agreement set. If we do not know the specific forms of the utility functions $\left(u^{i}().\right)$ but we know that $u^{i} \in U$ for all $i$, we can construct the Minimal Agreement Set $S(U)$ such that each element of $S(U)$ is a pair of lotteries, say $g^{k}($.$) and g^{h}($.$) ,$ and $g^{k}($.$) is unanimously preferred to g^{h}($.) by all the individuals so long their utility functions belong to the set $U$. Now we can look at the rule of stochastic dominance (relative to $U$ ) as the rule which is designed to identify the elements of $S(U)$. A contraction in $U$ results in a larger Minimal Agreement Set. We present our discussion on stochastic dominance and

\footnotetext{
* T Part of this research was carried out in the summer of 1987 when the author was visiting CORE (Louvain-la-Neuve, Belgium). The author wishes to thank Professor B. Cornet and the staff of CORE for their extended hospitality.
}

Originally published in Essays in Economic Analysis and Policy

(ed. D. Banerjee), Oxford University Press, 113-30 
comparative risk aversion in this framework, bearing in mind that the Minimal Agreement Set may not exist for certain choices of $U$ and $g($.) .

The literature on comparative risk aversion owes its origin to Pratt (1964). A general criterion for comparing risk aversion in the multivariate case was suggested by Khilstrom and Mirman (1974) restating a definition formulated originally in a two-state state-preference framework by Yaari (1969). The validity of the criterion in the two-commodity case was shown in Khilstrom and Mirman and was later generalized to the n-commodity case by Biswas (1983). In case of a general outcome set, including all certain and uncertain prospects, some risky prospects may not have a certainty equivalent included in the set. The reader may think of the outcome set being the union of several disjoint intervals on the real line. In such cases the study of comparative risk aversion becomes somewhat complicated. We have not dealt with this problem here, but we wish to make the readers aware of the problems involved in further generalization (see Nielsen 1986).

In this paper, we focus on the rules of stochastic dominance where $U$ contains the utility functions of individuals who are comparatively more risk averse than a reference individual $j$. Next, we study the case where $U$ is further contracted to include only the utility functions exhibiting declining comparative risk aversion with respect to $j$. The effort to relate the literature on stochastic dominance with that of comparative risk aversion is not entirely new. Meyer's (1977) work is an outstanding example. Actually, he worked out the condition for stochastic dominance with respect to comparatively risk averse individuals. In this paper, we have interpreted the condition in a different way by bringing in the concept of stochastic dominance in utility and extended the discussion to the case of declining comparative risk aversion. As the natural step towards conclusion, we have discussed the usefulness and limitations of the concept of stochastic dominance in utility in the multivariate case. Our concluding remarks centre around the importance, as well as the problems, of proving the existence of the Minimal Agreement Set in the context of any arbitrary set of risky alternatives.

\section{Rules For Stochastic Dominance And The Minimal Agreement Set}

A lottery is represented as a continuous probability density function $g^{1}(x) \in g(x), x \in w$, defined on an interval $w=[a, b] \in R^{1}$. The set $g(x)$ is the collection of all continuous probability functions. A generic Von Neumann-Morgenstern (VNM) utility function is a mapping, $u: w \rightarrow I_{0}$ where $I_{0}=[0,1]$. Any linear transformation of $u$ is also an admissible VNM utility function. Without any loss of generality, throughout this paper we shall only consider VNM utility functions in their generic forms. Consider the product space of two arbitrary lotteries,

Originally published in Essays in Economic Analysis and Policy

(ed. D. Banerjee), Oxford University Press, 113-30. 


\section{BISWAS Weak*Axiom of Independence}

$$
\Pi=\left\{g^{i}(x) \times g_{j}(x) \mid g^{i}(x), g^{j}(x) \in g(x), i \neq j\right\}
$$

Given a specific utility function $u^{1}$, let $S^{1}$ be a subset of $\Pi$ such that for any element $\left(g^{i}, g^{j}\right) \in S^{1} \subset \Pi, g^{i}$ is weakly preferable to $(R) g^{j}$. Clearly, $S^{1}$ includes all possible pairs of lotteries presented in a way such that the first element of the pair is weakly preferred to the second. ${ }^{1}$ It is also to be noted, throughout this paper we assume that the choice between lotteries is made in accordance with the expected utility hypothesis and the results of this paper are to be evaluated in that context.

Given another utility function $u^{2}$, let $S^{2}$ be defined in a similar way. Define $S\left(u^{1}, u^{2}\right)=S^{1} \cap S^{2}$. For two individuals with utility functions $u^{1}$ and $u^{2}$ respectively, $S\left(u^{1}, u^{2}\right)$ contains the pairs of lotteries such that the ranking of elements within a pair is the same for both individuals. In order to explain the construction of $S\left(u^{1}, u^{2}\right)$ let us assume that $g(x)$ contains only three lotteries, i.e. $g(x)=\left(g^{1}, g^{2}, g^{3}\right)$. This is different from what we assume elsewhere throughout this paper, namely that $g(x)$ contains all continuous lotteries. Here we assume this simply for the sake of convenience in demonstrating the construction of $S\left(u^{1}, u^{2}\right)$. Suppose there are two individuals with utility functions $u^{1}$ and $u^{2}$ respectively and their preference ordering over these three lotteries may be described as follows:

$$
\begin{gathered}
u_{1}(x): g^{1} R_{1} g^{2}, g^{2} R_{1} g^{3}, g^{1} R_{1} g^{3} \\
u_{2}(x): g^{2} R_{2} g^{1}, g^{2} R_{2} g^{3}, g^{1} R_{2} g^{3}
\end{gathered}
$$

The weak preference relationship for the $\mathrm{i}$-th individual is denoted by $R_{i}$ Since $R_{i}$ is generated by the expected utility, $R_{i}$ is transitive. In the context of the above example,

$$
S\left(u^{1}, u^{2}\right)=S^{1} \cap S^{2}=\left\{\left(g^{1}, g^{2}\right),\left(g^{2}, g^{3}\right),\left(g^{1}, g^{3}\right)\right\} \cap\left\{\left(g^{2}, g^{1}\right),\left(g^{2}, g^{3}\right),\left(g^{1}, g^{3}\right)\right\}=\left\{\left(g^{2}, g^{3}\right),\left(g^{1}, g^{3}\right)\right\}
$$

The above implies that so far as pairs $\left(g^{2}, g^{3}\right)$ and $\left(g^{1}, g^{3}\right)$ are concerned, both individuals prefer $g^{2}$ to $g^{3}$ and $g^{1}$ to $g^{3}$. What about the possibility of $S\left(u^{1}, u^{2}\right)$ being empty? For example, if $\tilde{u}^{2}(x): g^{2} R_{2} g^{1}, g^{3} R_{2} g^{2}, g^{3} R_{2} g^{1}$ then $S\left(u^{1}, \tilde{u}^{2}\right)=\varnothing$. Fortunately, this problem does not arise, if $g(x)$ contains all continuous lotteries (i.e. continuous probability density functions) and we impose some mild restrictions on utility functions. For example, if the utility functions $u^{1}(x)$ and $u^{2}(x)$ have positive first order derivatives, and $g(x)$ contains all continuous lotteries (which our example violates), then any pair of lotteries $\left(g^{1}, g^{2}\right)$ such that $\int_{a}^{x} g^{1}(x) \cdot d x \dagger \int_{a}^{x} g^{2}(x) \cdot d x$, will be included in $S\left(u^{1}, u^{2}\right)$. It is obvious that an infinite number of such lottery pairs are available. The above condition is known as the first order stochastic dominance rule (Quirk and Saposnik 1962). In all our subsequent discussions, we shall only

\section{Originally published in Essays in Economic Analysis and Policy}

(ed. D. Banerjee), Oxford University Press, 113-30. 
be concerned with classes of utility functions satisfying strict positive monotonicity, so that the agreement set $S\left(u^{1}, u^{2}\right)$ is non-empty.

Definition 1. Let $S(u)=S^{1} \cap S^{2} \cap \ldots \cap S^{n}, u=\left(u^{1}, u^{2} \ldots u^{n}\right) . S(u)$ is defined as the Agreed Set of Preference or, briefly the Agreement Set for a finite number of individuals $(i=1,2 \ldots n)$ with utility functions $u^{i}$.

In Definition 1, we assumed that individuals have specific and known utility functions, $u^{1}, u^{2} \ldots u^{n}$. Suppose the specific forms of the utility functions are not known but we know that they belong to a certain class of utility functions $U$. Then for any arbitrary selection of $n$ utility functions from $U$, we define the Minimal Agreement Set as follows.

Definition 2. $S(U)={ }_{u^{i} \in U} S^{i}$, is called the Minimal Agreement Set defined with respect to $U$. It is the intersection of all possible $S^{i}$ such that $u^{i}$ belongs to $U$. Note, the Minimal Agreement Set is valid for any arbitrary set of individuals whose utility functions belong to the set $U$.

$S(U)$ consists of those pairs of lotteries for which the preference within any pair is unanimously agreed upon by an arbitrary set of individuals for any arbitrary selection of their utility functions from $U$.

Theorem 1. The Minimal Agreement Set $S(U)$ satisfies the following properties:

i) $S(U)$ is a convex subset of $\Pi$

ii) $S\left(U^{\prime}\right) \subseteq S\left(U^{\prime \prime}\right)$ if $U^{\prime} \supseteq U^{\prime \prime}$

Proof. Consider two arbitrary pairs of lotteries $\left(g^{1}, g^{2}\right),\left(g^{3}, g^{4}\right) \in S(U)$. By construction, $g^{1} \bar{R} g^{2}$ and $g^{3} \bar{R} g^{4}$ where $\bar{R}$ symbolizes the weak preference unanimously agreed by each individual. Now,

$g=\lambda\left(g^{1}, g^{2}\right)+(1-\lambda) \cdot\left(g^{3}, g^{4}\right)=\left\{\left(\lambda g^{1}+(1-\lambda) g^{3}\right),\left(\lambda g^{2}+(1-\lambda) g^{4}\right)\right\}=\left(g^{5}, g^{6}\right), 0 \dagger \lambda \dagger 1$

$g^{5}$ is equivalent to a compound lottery with $\lambda$ probability of holding $g^{1}$ and $(1-\lambda)$ probability of holding $g^{3}$. Similarly, $g^{6}$ is equivalent to a compound lottery with $\lambda$ probability holding $g^{2}$ and $(1-\lambda)$ probability of holding $g^{4}$. Since $g^{1} \bar{R} g^{2}$ and $g^{3} \bar{R} g^{4}$, by the expected utility hypothesis, $g^{5} \bar{R} g^{6}$, i.e. $g=\left(g^{5}, g^{6}\right) \in S(U)$.

Originally published in Essays in Economic Analysis and Policy

(ed. D. Banerjee), Oxford University Press, 113-30. 


\section{BISWAS Weak*Axiom of Independence}

ii) The second property follows from the definition of the Minimal Agreement Set. Since ${u^{i} \in u^{\prime}} s^{i} \subseteq \underset{u^{i} \in u^{\prime \prime}}{\cap} s^{i}$ for $u^{\prime} \supseteq u^{\prime \prime}$, by definition $S\left(u^{\prime}\right) \subseteq s\left(u^{\prime \prime}\right)$.

(QED)

The minimal agreement set is largest when $U$ is a singleton. This is the case when all individuals have the same utility function so that $S(U)$ includes all possible pairs of lotteries arranged in a way that the first element in the pair is weakly preferred to the second element by everyone.

Consider two lotteries $g^{1}, g^{2}$ and a set of $n$ individuals each of whom have utility functions belonging to a set $U$. How can we test that the ranking (ordering) of these two lotteries will be unanimously agreed by all individuals? In other words, how can we characterize a pair of lotteries $\left(g^{1}, g^{2}\right)$ such that $\left(g^{1}, g^{2}\right) \in S(U)$ ? This is where the rules of stochastic dominance becomes important. Let $U^{1}$ be the set of utility functions having positive first order derivatives defined everywhere in the interior of $w=[a, b]$. If $\left(g^{1}, g^{2}\right)$ satisfies the first order stochastic dominance rule (FSD), i.e.

$$
\int_{a}^{x} g^{1}(x), d x \dagger \int_{a}^{x} g^{2}(x) \cdot d x, \forall x \in w
$$

then $\left(g^{1}, g^{2}\right) \in S\left(U^{1}\right)$. As noted earlier, this result follow from Quirk and Saposnik (1962). In a similar way, if a pair of lotteries $\left(g^{1}, g^{2}\right)$ satisfy the second order stochastic dominance conditions (SSD), i.e.

$$
\int_{a}^{y} \int_{a}^{y} g^{1}(x) \cdot d x \cdot d y \dagger \int_{a}^{y} \int_{a}^{y} g^{2}(x) \cdot d x \cdot d y \quad \forall x, y \in w
$$

then $\left(g^{1}, g^{2}\right) \in S\left(U^{2}\right)$ where $U^{2}$ is the set of twice differentiable concave utility functions with positive first order derivatives. This result follows from Hadar and Russell (1969).

Definition 3. Given a rule of stochastic dominance $(r)$, the set of lottery-pairs $\left(g^{i}, g^{j}\right) \in \Pi, i \neq j$, which satisfy the rule with $g^{i} \bar{R} g^{j}$, is called the admissible set of lotterypairs under the rule, or, simply the admissible set, $A(r)$.

Remark 1. Given a set of permissible utility functions $U$, if a rule is both necessary and sufficient for stochastic dominance with respect to all individuals having $u \in U$, then $A(r)=S(U)$. If the rule is only necessary then $A(r) \supset S(U)$. If the rule is only sufficient then $A(r) \subset S(U)$. Since the FSD and SSD rules are both necessary and sufficient, $A(F S D)=S\left(U^{1}\right)$ and $A(S S D)=S\left(U^{2}\right)$.

Originally published in Essays in Economic Analysis and Policy

(ed. D. Banerjee), Oxford University Press, 113-30. 
Remark 2. The admissible set under the second order stochastic dominance rule includes the admissible set under the first order stochastic dominance rule. This can be shown in the following way. $U^{2} \subseteq U^{1} \rightarrow S\left(U^{2}\right) \supseteq S\left(U^{1}\right)$. Since, $A(F S D)=S\left(U^{1}\right)$ and $A(S S D)=S\left(U^{2}\right)$, being necessary and sufficient rules, $A(S S D) \supseteq A(F S D)$.

We have already noted, $S\left(U^{1}\right) \neq \varnothing$. Therefore, by Theorem $1, S\left(U^{t}\right) \neq \varnothing$ for any $U^{t} \subseteq U^{1}$. In other words, for any arbitrary class of differentiable monotonic increasing utility functions, the minimal agreement set is non-empty. The structure of this set will depend upon the properties of the class of functions $U^{t}$. The problem is to define a rule $\left(r^{t}\right)$ such that the admissible set under the rule, $A\left(r^{t}\right)$, coincides with $S\left(U^{t}\right)$. The principal challenge of research in the area of stochastic dominance is to find a rule in the context of a reasonable class of utility functions such that $A\left(r^{t}\right) \subseteq S\left(U^{t}\right)$. For example Whitmore (1970) considered the set of utility functions $\left(U^{3}\right)$ satisfying decreasing absolute risk aversion. He found the necessary and sufficient conditions as,

i) $\quad \int_{a}^{z} \int_{a}^{y}\left(G^{1}(x)-G^{2}(x)\right) d x . d y \dagger 0, x, y, z \in w$ where $G^{i}(x)=\int_{a}^{x} g^{i}(x) \cdot d x$.

ii)

$$
\int_{a}^{b}\left(G^{1}(x)-G^{2}(x)\right) d x \dagger 0
$$

Since we are restricting the freedom of individuals by forcing them to choose from a smaller set of utility functions, the minimal agreement set contains more lottery-pairs, i.e., $S\left(U^{1}\right) \subseteq S\left(U^{2}\right) \subseteq S\left(U^{3}\right)$.

In the next section, we shall consider the question of stochastic dominance for a class of utility functions which has recently attracted a lot of attention, namely, the class of utility functions which are concave transformations of a particular concave utility function, $u^{j}(x)$.

$$
U^{4}=\left\{u \mid u=v\left(u^{j}(x), v^{\prime}>0, v^{\prime \prime} \dagger 0, \quad u^{j}(x) \text { is weakly concave in } x \in w\right\} .\right.
$$

The economic interpretation of $U^{4}$ is that any individual $i$ with $u^{i} \in U^{4}$ and $u^{i} \neq u^{j}$, is comparatively more risk averse than an individual with $u^{j}$ as his utility function. The concept of comparative risk aversion, introduced by Pratt (1964) has recently attracted considerable attention. In the next section, we shall examine a rule of stochastic dominance which is compatible with unanimous ordering of lotteries by a group of individuals who are comparatively more risk averse than a certain individual $j$.

\section{Stochastic Dominance in Utility and Comparative Risk Aversion}

\section{Originally published in Essays in Economic Analysis and Policy}

(ed. D. Banerjee), Oxford University Press, 113-30. 


\section{BISWAS Weak*Axiom of Independence}

Consider the set $U^{1}$ which includes all differentiable utility functions with positive first order derivatives. For $u^{1}, u^{2} \in U^{1}$, representing the utility functions of individuals 1 and 2 respectively, we may write $u^{2}=v\left(u^{1}\right), v^{\prime}>0$. Let $u_{i}^{-j}$ represent the expected utility of individual $j$ from lottery $i$. By using integration by parts, the following relation can be easily established:

$$
\begin{aligned}
u_{1}^{-j} & -u_{2}^{-j}=\int_{a}^{b} u^{j}(x)\left[g^{1}(x)-g^{2}(x)\right] \cdot d x \\
& =-\int_{a}^{b}\left[G^{1}(x)-G^{2}(x)\right] \cdot d x
\end{aligned}
$$

where $G^{i}(x)=\int_{a}^{b} g^{i}(x) \cdot d x$

The expected utility maximizing individual weakly prefers $g^{1}(x)$ to $g^{2}(x)$ if and only if $\int_{a}^{b}\left[G^{1}(x)-G^{2}(x)\right] \cdot d u^{j} \dagger 0$. Since $u^{j}(x)$ and $x$ have one-to-one correspondence $\left(u^{j}(x)\right.$ being a monotonic, increasing function) we may define a lottery also as a probability density function on the utility space. Let $H^{i}\left(u^{j}\right)$ be the probability that if lottery $i$ is chosen by individual $j$, he will end up with utility not more than $u^{j}$. By definition $H^{i}\left(u^{j}\right)=G^{i}\left(x\left(u^{j}\right)\right)$

From (1) the next lemma follows immediately.

Lemma 1. Let $g^{1}\left(u^{j}\right)$ and $g^{2}\left(u^{j}\right)$ be two lotteries defined on the $j$-th individual's utility space $I_{0}=[0,1]$. Individual $j$ will prefer $g^{1}$ to $g^{2}$ if and only if,

$$
\int_{0}^{1} H^{1}\left(u^{j}\right) d u^{j} \leq \int_{0}^{1} H^{2}\left(u^{j}\right) d u^{j}, \quad u_{j} \in I_{0}
$$

Note, the limits of integration have changed from [a,b] to [0,1] in comparison with (1) because $u^{j}: w \rightarrow I_{0}$ and $d u^{j}>0$.

Definition 4. Lottery 1 has first order stochastic dominance in utility (FSDU) over lottery 2 for individual $j$, if and only if $H^{1}\left(u^{j}\right) \dagger H^{2}\left(u^{j}\right) \forall u \in I_{0}$.

Definition 5. Lottery 1 has second order stochastic dominance in utility over lottery 2 for individual $j$ if and only if,

$$
\int_{0}^{u} H^{1}\left(u^{j}\right) \cdot d u^{j} \dagger \int_{0}^{u} H^{2}\left(u^{j}\right) \cdot d u^{j}, \quad \forall u \in I_{0}
$$

Originally published in Essays in Economic Analysis and Policy

(ed. D. Banerjee), Oxford University Press, 113-30. 
It is well known that FSD implies SSD. Similarly, it can easily be seen that FSDU implies SSDU. Since utility functions are monotonic and increasing, FSDU implies FSD and vice versa. Next we show that SSD implies SSDU if the utility function is weakly concave $\left(d u^{j} / d x>0, d^{2} u^{j} / d x^{2} \dagger 0\right)$ but the converse is not true.

Theorem 2. If a lottery $g^{1}(x)$ has second order stochastic dominance (SSD) over $g^{2}(x)$, then for any weakly risk averse individual $j, g^{1}(x)$ has second order stochastic dominance in utility (SSDU) over $g^{2}(x)$. The converse is not true.

Proof. Consider $D\left(\hat{u}^{j}\right)=\int_{0}^{\hat{u}^{j}}\left[H^{1}\left(u^{j}\right)-H^{2}\left(u^{j}\right)\right] d u^{j}$

$$
\begin{aligned}
& =\int_{a}^{\hat{x}}\left[H^{1}\left(u^{j}(x)\right)-H^{2}\left(u^{j}(x)\right)\right]\left(d u^{j} / d x\right) \cdot d x \text { where } \hat{u}^{j}=u^{j}(\hat{x}) \\
& =\int_{a}^{\hat{x}}\left[G^{1}(x)-G^{2}(x)\right]\left(d u^{j} / d x\right) \cdot d x
\end{aligned}
$$

Integrating by parts,

$$
\begin{aligned}
& D\left(\hat{u}^{j}\right)=\left[\left(d u^{j} / d x\right) \int_{a}^{x}\left(G^{1}(y)-G^{2}(y)\right) \cdot d y\right]_{a}^{\hat{x}} \\
& -\int_{a}^{\hat{x}}\left(d^{2} u^{j} / d x^{2}\right) \int_{a}^{x}\left[G^{1}(y)-G^{2}(y)\right] d y d x
\end{aligned}
$$

We used the notation $y$ instead of $x$ in order to avoid confusion between the variable and the limits. Second order stochastic dominance of $g^{1}(x)$ over $g^{2}(x)$ implies that,

$$
C(x)=\int_{a}^{x}\left(G^{1}(y)-G^{2}(y)\right) d x \dagger \mathbf{0} \forall x \in w
$$

Since $\left(d u^{j} / d x\right)>0$ and $d^{2} u^{j} / d x^{2} \dagger 0$, it is clear from (2) that $D\left(\hat{u}^{j}\right) \dagger 0$ for any $\hat{u}^{j} \in I_{0}$, if the SSD rule holds. But the converse is not true. Even if for some values of $x, C(x)>0$, $D\left(u^{j}\right)$ may be non-positive for all $\hat{u}^{j} \in I_{0}$. Although this should be apparent from (2), it may be worthwhile to provide an example.

Consider an utility function $u^{j}, d u^{j}>0, d^{2} u^{j}<0$ and a partition of $w$ such that $w_{1}=[a, b-\delta]$ and $w_{2}=[b-\delta, b]$ where $\delta$ is a small positive value. Let us assume

$$
\begin{aligned}
& C(x)=0 \text { when } x=a \\
& C(x)<0 \text { when } a<x<b-\delta
\end{aligned}
$$

Originally published in Essays in Economic Analysis and Policy

(ed. D. Banerjee), Oxford University Press, 113-30. 


$$
\begin{aligned}
& C(x)=0 \text { when } x=b-\delta \\
& 0<C(x)<K_{0} \text { when } b-\delta<x \dagger b
\end{aligned}
$$

Rewriting (2) as

$$
D\left(\hat{u}^{j}\right)=D\left(u^{j}(\hat{x})\right)=\left[\left(d u^{j} / d x\right) \cdot C(x)\right]_{a}^{\hat{x}}-\int_{a}^{\hat{x}}\left(d^{2} u^{j} / d x^{2}\right) \cdot C(x) \cdot d x
$$

It is clear that, $D\left(u^{j}(0)\right)=0$ and $D\left(u^{j}(\hat{x})\right)<0$ for $0<\hat{x} \leqq b-\delta$.

That is, for $x \in w_{1}, D\left(u^{j}(\hat{x})\right) \leqq 0$ and $D\left(u^{j}(b-\delta)\right)=K_{1}<0$.

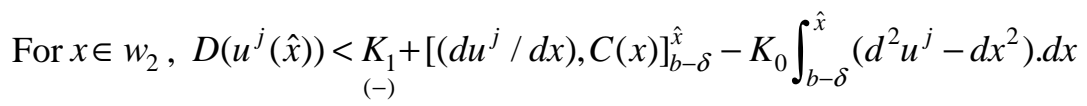

$$
\begin{aligned}
& =\underset{(-)}{K_{1}}+\left[\left(d u^{j} / d x\right)\left(C(x)-K_{0}\right)\right]_{b-\delta}^{X}
\end{aligned}
$$

By construction, $0 \leqq\left(C(x)-K_{0}\right) \leqq K_{0}$ for $b-\delta \leqq x \leqq b$. Then given (4), we can always construct the utility function $u^{j}$ with $d u^{j} / d x$ so small in the regions of $b-\delta<x \leqq b$, that for $x \in w_{2}, D\left(u^{j}(\hat{x})\right)<0$. For such a utility function $D\left(u^{j}\right) \leqq 0$ for all $u^{j} \in I_{0}$ but $C(\hat{x})>0$ for $x \in w_{2}$, violating the SSD rule.

(QED)

The relationship between different types of stochastic dominance discussed so far, may be represented as follows:

$$
\begin{gathered}
\mathrm{FSD} \rightarrow \mathrm{SSD} \\
\text { FSDU } \rightarrow \text { SSDU }
\end{gathered}
$$

Two important features of SSDU are to be carefully noted. First, it is weaker than SSD in the sense that it has a larger admissible set, $\mathrm{A}$ (SSD) $\subset \mathrm{A}$ (SSDU). Second, it is dependent on the utility function of a particular individual $j$. Next, consider two weakly risk averse individuals $j$ and $k$ with $u^{j}$ and $u^{k} \in U^{2}$. As noted earlier, we may write $u^{k}=v\left(u^{j}\right), v^{\prime}>0$. Following Pratt (1964) and others (see, Khilstrom and Mirman 1974; Biswas 1983), we may say that $k$ is comparatively more risk averse than $j$ if $v^{\prime \prime} \leqq 0$ for all $u^{j} \in I_{0}$. Given a lottery, $k$ is willing to pay a higher risk premium than that of $j$.

Theorem 3. Consider a weakly risk averse individual $j$ with utility function $u^{j} \in U^{2}$ and a class of utility functions $U^{4}=\left\{u \mid u=v\left(u^{j}(x)\right), v^{\prime}>0, v^{\prime \prime} \leqq 0\right\}$. Every individual $k$ with

\section{Originally published in Essays in Economic Analysis and Policy}

(ed. D. Banerjee), Oxford University Press, 113-30. 
$u^{k} \in U^{4}$ weakly prefer $g^{1}$ to $g^{2}$ if and only if, $g^{1}$ has second order stochastic dominance in utility over $g^{2}$.

Proof. (Sufficiency) By (1) and lemma (1),

$$
u_{1}^{-k}-u_{2}^{-k}=-\int_{0}^{1} v^{\prime}\left(H^{1}\left(u^{j}\right)-H^{2}\left(u^{j}\right)\right) d u^{j}
$$

Integrating by parts,

$$
\begin{aligned}
u_{1}^{-k}-u_{2}^{-k}=- & {\left[v^{\prime} \int_{0}^{u^{j}}\left(H^{1}(y)-H^{2}(y)\right) d y\right]_{0}^{1}+\int_{0}^{1} v^{\prime \prime} \int_{0}^{u^{j}}\left(H^{1}(y)-H^{2}(y)\right) d y d u^{j}=-\left[v^{\prime} D\left(u^{j}\right)\right]_{0}^{1}+\int_{0}^{1} v^{\prime \prime} . D\left(u^{j}\right) d u^{j} } \\
& \text { where } D\left(u^{j}\right)=\int_{0}^{u^{j}}\left(H^{1}(y)-H^{2}(y)\right) d y
\end{aligned}
$$

If $g^{1}$ has SSDU over $g^{2}$ for individual $j, D\left(u^{j}\right) \leqq 0$ for all $u^{j} \in I_{0}$.

Now,

$$
\left[v^{\prime} D\left(u^{j}\right)\right]_{0}^{1}=v^{\prime}(1) . D(1)-v^{\prime}(0) \cdot D(0) \text { and, }
$$

i) $D(1) \leqq D(0)=0$ because $D(1) \leqq 0$

ii) $v^{\prime}(1) \leqq v^{\prime}(0)$ because $v^{\prime \prime} \leqq 0$

Therefore, $\left[v^{\prime} D\left(u^{j}\right)\right]_{0}^{1} \leqq 0$

Again, $\int_{0}^{1} v^{\prime \prime} D\left(u^{j}\right) d u^{j} \geqq 0$, because $v^{\prime \prime} \leqq 0$ and $D\left(u^{j}\right) \leqq 0$. So,

$$
u_{1}^{-k}-u_{2}^{-k}=-\left[v^{\prime} D\left(u^{j}\right)\right]_{0}^{1}=\int_{0}^{1} v^{\prime \prime} \cdot D\left(u^{j}\right) \cdot d u^{j} \geqq 0
$$

\section{(Necessity)}

Let $\int_{0}^{u^{j}}\left(H^{1}(y)-H^{2}(y)\right) d y>0$ for $u^{j} \in I=(m, n) \subset I_{0}$

We can always construct a concave utility function $u^{k}=v\left(u^{j}\right)$ such that $v^{\prime}\left(u^{j}\right)=c_{0}>0$ for $u^{j} \in[0, m], v^{\prime \prime}\left(u^{j}\right)<0$ for $u^{j} \in I$ and $v^{\prime}\left(u^{j}\right)=c_{1}, 0<c_{1}<c_{0}$ for $u^{j} \in[n, 1]$. Since $u^{j} \in U^{4}$,

Originally published in Essays in Economic Analysis and Policy

(ed. D. Banerjee), Oxford University Press, 113-30. 
individual $j$ prefers $g^{1}$ to $g^{2}$. Therefore by lemma $1, \int_{0}^{1}\left[H^{1}\left(u^{j}\right)-H^{2}\left(u^{j}\right)\right] d u^{j} \leqq 0$. Since by construction, $v^{\prime}(0)=c_{0}>0$,

$$
-\left[v^{\prime} \int_{0}^{u^{j}}\left[H^{1}\left(u^{j}\right)-H^{2}\left(u^{j}\right)\right] d u^{j}\right]_{0}^{1}=-c_{1} \int_{0}^{1}\left[H^{1}\left(u^{j}\right)-H^{2}\left(u^{j}\right)\right] d u^{j} \geqq 0
$$

Noting $v^{\prime \prime} \leqq 0$ and particularly $v^{\prime \prime}=0$ for $u^{j} \in\left(I_{0}-I\right)$, by (5),

$u_{1}^{-k}-u_{2}^{-k}=-c_{1} \int_{0}^{1}\left[H^{1}\left(u^{j}\right)-H^{2}\left(u^{j}\right)\right] d u^{j}+\int_{m}^{n} v^{n} \int_{0}^{u^{j}}\left[H^{1}(y)-H^{2}(y)\right] d y d u^{j}$

The first term in (6) is non-negative by lemma 1 . Since by construction $v^{\prime \prime}<0$ over the range $(m, n)$ and $\int_{0}^{u^{j}}\left(H^{1}(y)-H^{2}(y)\right) d y>0$ for $u^{j} \in(m, n)$, the second term in (6) is strictly negative. Now, we can modify our function $v\left(u^{j}\right)$ by choosing $c_{1}$ arbitrarily small so that by (6), $u_{1}^{-k}-u_{2}^{-k}<0$, i.e., the individual $k$ prefers $g^{2}$ to $g^{1}$. The same kind of contradiction may be reached when SSDU is violated over any countable union of intervals.

(QED)

Theorem 3 bridges an important gap between the literature on comparative risk aversion and the literature on rules for stochastic dominance. From theorem 2, we know that SSDU is a broader rule than SSD in the sense that $\mathrm{A}(\mathrm{SSDU}) \supset \mathrm{A}(\mathrm{SSD})$. As expected by theorem 1 , the class of utility functions $U^{4}$, for which it is both a necessary and a sufficient rule, is a subset of $U^{2}$. The following result is worth mentioning.

Theorem 4. If $j$ is a risk neutral individual (extreme case of weak risk aversion) with utility function $u^{j}=t . x, t>0$, for whom $g^{1}$ has SSDU over $g^{2}$, then all risk averse individuals will prefer $g^{1}$ over $g^{2}$.

Proof. If $u^{j}=t . x, t>0$, then by (2) in theorem 2,

$$
\begin{gathered}
D\left(\hat{u}^{j}\right)=\left[t \int_{a}^{x}\left(G^{1}(y)-G^{2}(y)\right) d y\right]_{a}^{\hat{x}}= \\
t \int_{a}^{\hat{x}}\left(G^{1}(y)-G^{2}(y)\right) d y
\end{gathered}
$$

SSDU implies $D\left(\hat{u}^{j}\right) \dagger 0$ for all $\hat{u}^{j} \in I_{0}$. Since there is one to one correspondence between $u^{j}$ and $x$, by (7), SSDU implies,

Originally published in Essays in Economic Analysis and Policy

(ed. D. Banerjee), Oxford University Press, 113-30. 


$$
\int^{\hat{x}} \alpha\left(G^{1}(y)-G^{2}(y)\right) d y \leqq 0 \text { for all } \hat{x} \in w .
$$

This is precisely the SSD rule. Therefore, if $g^{1}$ has SSDU over $g^{2}$ with respect to a risk neutral individual, then $g^{1}$ has SSD over $g^{2}$. This implies, all risk averse individuals prefer $g^{1}$ over $g^{2}$.

(QED)

This theorem is helpful in explaining why in another sense SSDU is a more general rule than SSD. If the (reference) individual $j$ is risk neutral, then $U^{4}$ comprises of all (weakly) risk averse individuals and the SSDU rule coincides with the SSD rule. Therefore, SSD rule may be considered to be an application of the SSDU rule in a special case.

\section{Stochastic Dominance and Declining Comparative Risk Aversion}

Consider a group of individuals who are comparatively more risk averse than a particular (weakly) risk averse individual $j$. The additional feature of this group is that the difference in the degree of (absolute) risk aversion between any member of the group and the individual $j$ declines with increases in wealth $x$. This is quite a reasonable group characteristic. At a lower level of wealth one may be strikingly more risk averse than another, but when both are put in a higher wealth bracket, the difference in the degree of risk aversion is likely to be smaller and may even be negligible.

Theorem 5. Let $u^{k}$ represent the monotonic increasing utility function of a risk averse individual who is strictly more risk averse than another (weakly) risk averse individual $j$ with the characteristic that the difference in the absolute degree of risk aversion declines with increases in $x \in w$. Then $u^{k}$ may be expressed as a function of $u^{j}$ satisfying the following relationship:

$$
u^{k}=v\left(u^{j}\right), v^{\prime}>0, v^{\prime \prime}<0, v^{\prime} ">0
$$

Proof. $v^{\prime}>0$ because the utility functions of both the individuals are monotonic and increasing. $v^{\prime \prime}<0$ because $k$ is strictly more risk averse than $j$. We prove that $v^{\prime} ">0$.

$$
\begin{array}{ll}
\text { Using } & u^{k}=v\left(u^{j}\right), v^{\prime}>0, v^{\prime \prime}<0 \text { for } u^{j} \in I_{0}, \\
& d u^{k} / d x=v^{\prime}\left(d u^{j} / d x\right) \\
& d^{2} u^{k} / d x^{2}=v^{\prime}\left(d^{2} u^{j} / d x^{2}\right)+v^{\prime \prime}\left(d u^{j} / d x\right)^{2}
\end{array}
$$

Originally published in Essays in Economic Analysis and Policy

(ed. D. Banerjee), Oxford University Press, 113-30. 


\section{BISWAS Weak*Axiom of Independence}

Therefore, $R_{k}=R_{j}-\left(v^{\prime \prime} / v^{\prime}\right)\left(d u^{j} / d x\right)$

Where $R_{i}$ is the degree of absolute risk aversion for the $i$-th individual $(i=j, k)$. Since $v^{\prime}>0$ and $v "<0, R_{k}>R_{j}$. Declining comparative risk aversion implies that,

$$
\frac{d}{d x}\left[\frac{v^{\prime \prime}}{v^{\prime}} \cdot \frac{d u^{j}}{d x}\right]>0
$$

The above relationship requires $v^{\prime} ">0$

The class of utility functions exhibiting declining comparative risk aversion in comparison with $u^{j}$ is defined as,

$$
U^{5}=\left\{u / u=v\left(u^{j}\right), v^{\prime}>0, v^{\prime \prime}<0 \text { and } \frac{d}{d x}\left[\frac{v^{\prime \prime}}{v^{\prime}} \cdot \frac{d u^{j}}{d x}\right]>0\right\}
$$

We assume that derivatives of $v$ up to the third order are continuous in $u^{j}$.

Theorem 6. Consider a weakly risk averse individual $j$ with utility function $u^{j} \in U^{2}$ and the class of utility function $U^{5}$ associated with $u^{j}$. Each individual $k$ with $u^{k} \in U^{5}$, weakly prefer $g^{1}$ to $g^{2}$ if and only if,

(i) $\int_{0}^{1}\left[H^{1}\left(u^{j}\right)-H^{2}\left(u^{j}\right)\right] d u^{j} \leqq 0$, and,

(ii) $\int_{0}^{u^{j}} \int_{0}^{z}\left[H^{1}(y)-H^{2}(y)\right] d y d z \leqq 0$ for all $u^{j} \in I_{0}$.

Proof. Before proceeding to the details of the proof, we note that by lemma 1, condition (i) simply means, individual $j$ prefers $g^{1}$ to $g^{2}$. Since $u^{j} \notin U^{5}$, because $U^{5}$ contains only (strictly) declining comparatively risk averse individuals, we had to bring in condition (i). In this section, we are considering a narrower class of utility functions because strictly declining comparative risk aversion is presumed to have an appeal to economic intuition. If we assume the weaker case of $v^{\prime \prime} \leqq 0$ and non-increasing comparative risk aversion, condition (i) is eliminated because $u^{j} \in U^{5}$.

A. (Sufficiency) By integrating the second term in (5)

$$
u_{1}^{-k}-u_{2}^{-k}=-\left[v^{\prime}\left(u^{j}\right) \int_{0}^{u^{j}}\left[H^{1}(y)-H^{2}(y)\right] d y\right]_{0}^{1}+
$$

Originally published in Essays in Economic Analysis and Policy

(ed. D. Banerjee), Oxford University Press, 113-30. 


$$
\begin{aligned}
& {\left[v^{\prime \prime}\left(u^{j}\right) \int_{0}^{u^{j}} \int_{0}^{z}\left(H^{1}(y)-H^{2}(y)\right) d y d z\right]_{0}^{1}-} \\
& \int_{0}^{1} v^{\prime \prime}\left(u^{j}\right) \int_{0}^{u^{j}} \int_{0}^{z}\left(H^{1}(y)-H^{2}(y)\right) d y d z
\end{aligned}
$$

Since condition (i) holds, by applying the same kind of reasoning as in theorem 3 , we can show that the first term in (8) is non-negative. Again, since $v^{\prime \prime}<0$ and condition (ii) holds, we can use a similar reasoning to show that the second term is non-negative. Since $v^{\prime \prime}>0$ and condition (ii) holds, the third term is obviously non-negative. Therefore $u_{1}^{-k}-u_{2}^{-k} \geqq 0$.

B. (Necessity) The proof for necessity is divided into two parts. First, we prove that condition (i) is necessary. Second, we show that given condition (i), condition (ii) is also necessary. Condition (i) is necessary by the continuity argument. To be explicit, suppose $\int_{0}^{1}\left(H^{1}\left(u^{j}\right)-H^{2}\left(u^{j}\right)\right) d u^{j}>0$. Now think of $u^{\sim k}=\tilde{v}\left(u^{j}\right)$ where $\tilde{v}^{\prime}=q$ (a positive constant).

Apparently,

$$
\int_{0}^{1} \tilde{v}^{\prime}\left(H^{1}\left(u^{j}\right)-H^{2}\left(u^{j}\right)\right) d u^{j}=-\left(\overline{\tilde{u}}^{k}-\overline{\tilde{u}}^{k}\right)>0
$$

i.e. $k$ prefers $g^{2}$ to $g^{1}$. We can always construct a function $u^{k}=v\left(u^{j}\right)$ such that $u^{k}$ is extremely close to $u^{\sim k}=q \cdot u^{j}$ with $v^{\prime \prime}<0$ and $v^{\prime} ">0$ (via small perturbations from $v^{\prime \prime}=0$ and $\left.v^{\prime \prime}=0\right)$ such that $\int_{0}^{1} v^{\prime}\left(H^{1}\left(u^{j}\right)-H^{2}\left(u^{j}\right)\right) d u^{j}=-\left(u_{1}^{-k}-u_{2}^{-k}\right)>0$. Though $u^{k} \in U^{5}$ $k$ prefers $g^{2}$ to $g^{1}$ which contradicts our assumption. Hence $\int_{0}^{1}\left(H^{1}\left(u^{j}\right)=H^{2}\left(u^{j}\right)\right) d u^{j}$ must be non-positive.

To show that condition (ii) is also necessary, we use the same kind of argument used in the necessity part of theorem 3. Let a pair of lotteries $g^{1}$ and $g^{2}$ be such that

$$
\psi\left(u^{j}\right)=\int_{0}^{u^{j}} \int_{0}^{z}\left(H^{1}(y)-H^{2}(y)\right) d y d z>0 \text { for } u^{j} \in I=(m, n) \quad I_{0}=[0,1]
$$

Then consider a utility function $u^{k}=v\left(u^{j}\right)$ such that $v^{\prime}>0$ and,

(a) $v^{\prime \prime}\left(u^{j}\right)=c_{0}<0$ for $u^{j} \in[0, m]$

(b) $v^{\prime \prime}\left(u^{j}\right)<0$ and $v^{\prime} "\left(u^{j}\right)>0$ for $u^{j} \in I=(m, n)$

Originally published in Essays in Economic Analysis and Policy

(ed. D. Banerjee), Oxford University Press, 113-30. 


$$
\text { (c) } v^{\prime}\left(u^{j}\right)=c_{1}, 0>c_{1}>c_{0} \text { for } u^{j} \in[n, 1]
$$

By (a), $v^{\prime}(0)$ is finite and therefore,

$$
-\left[v^{\prime}\left(u^{j}\right) \int_{0}^{u^{j}}\left(H^{1}(y)-H^{2}(y)\right) d y\right]_{0}^{1}=-v^{\prime}(1) \int_{0}^{1}\left[H^{1}(y)-H^{2}(y)\right] d y>0
$$

By condition (i) of this theorem which has been shown to be necessary, (8) may now be written as,

$$
u_{1}^{-k}-u_{2}^{-k}=-v^{\prime}(1) \int_{0}^{1}\left(H^{1}\left(u^{j}\right)-H^{2}\left(u^{j}\right)\right) d u^{j}+c_{1} \psi(1)-c_{0} \psi(0)-\int_{m}^{n} v^{\prime \prime}\left(u^{j}\right) \psi\left(u^{j}\right) d u^{j}
$$

Given our hypothesis that $\psi\left(u^{j}\right)>0$ for $u^{j} \in(m, n)$ and $v^{\prime} ">0$ (by construction), the third term in (9) is negative. We can always choose $v^{\prime}(1), c_{1}$ and $c_{0}$ so small that $u_{1}^{-k}-u_{2}^{-k}<0$. Therefore, if $\psi\left(u^{j}\right)>0$ for $u^{j} \in(m, n)$, there exists $u^{k} \in U^{5}$ such that $u_{1}^{-k}-u_{2}^{-k}<0$. This completes the necessity part of the proof.

(QED)

The conditions (i) and (ii) are generalizations of Whitmore's (1970) conditions for third order (degree) stochastic dominance. If the individual $j$ is a risk neutral person, then $U^{5}$ contains all utility functions with strictly decreasing risk aversion. As expected, one can easily show that in this particular case, conditions (i) and (ii) reduce to Whitmore's conditions. However, in theorem 6, the weakly risk averse individual $j$ may, over a range of $x$, even exhibit increasing risk aversion and consequently $U^{5}$ contain utility functions $u^{k}$ which may exhibit increasing risk aversion somewhere in that range. Therefore, the class of utility functions $U^{5}$, need not be a subset of the class of utility functions exhibiting declining risk aversion.

\section{Multivariate Extensions}

For a better appreciation of the concept of stochastic dominance, we are required to extend our discussion to the n-variables case. Obviously, given the utility function of our referenceindividual $j$ (and hence the set of indifference or iso-utility curves) we may formally define FSDU and SSDU with respect to $u^{j}$ as previously. However, in the multivariate case, FSDU with respect to a particular utility function $u^{j}$ does not imply FSD. Since FSD is associated with the choice of $U^{1}$ as the set of all possible utility functions, we have to take into account all possible sets of non-negatively sloped indifference curves for the reference individual.

Define a subset $S$ of $w^{n}$ as a comprehensive set if $b \in S$ and $a \in w^{n}, a \leqq b$ imply $a \in S$. The set $w^{n}$, on which the lotteries are defined, is a compact and convex subset of $R^{n}$. According to Levhari, Paroush and Peleg (1975), FSD in the multivariate case requires that

\section{Originally published in Essays in Economic Analysis and Policy}

(ed. D. Banerjee), Oxford University Press, 113-30. 
Review of Economic Analysis 4 (2012) 105-122

$\int_{S}\left(g^{1}(x)-g^{2}(x)\right) d x<0$ for all comprehensive subsets $S \in w^{n}$. Loosely speaking, the area below any negatively sloped indifference curve is a comprehensive set. Therefore, the above requirement asserts that if $g^{1}(x)$ has FSDU over $g^{2}(x)$ for all $u^{j} \in U^{1}$ then $g^{1}(x)$ has FSD over $g^{2}(x)$. The multivariate extension of SSD is much more complicated. The interested reader is referred to Russell and Seo (1978).

As we have already noted, we may formally define FSDU and SSDU in the multivariate case as we did in the univariate case. But these concepts have operational meaning only if we consider a group of individuals with utility functions $\quad\left\{u^{k}\right\}$ such that $u^{k}=v\left(u^{j}\right)$. In other words, we are restricted to the case of individuals having different generic VNM utility functions but the same set of underlying indifference curves. However, this is not an additional restriction if we remember that in the multivariate case, this condition must be satisfied when comparing the degree of risk aversion among individuals in a group. The reader is referred to Khilstrom and Mirman (1974), Biswas (1983) and Karni (1985, ch. 6). If the underlying preference orderings on $w^{n}$ are same for all individuals, we can write $u^{k}=v\left(u^{j}\right), v^{\prime}>0$. If $k$ is more risk averse than $j$, then $v^{\prime \prime}<0$ and declining comparative risk aversion requires $v^{\prime} ">0$. Since the conditions of stochastic dominance in utility are stated in the utility space, it is clear that theorems 3 and 6 can easily be extended to the multivariate case provided individuals have identical preference orderings defined on $w^{n}$ to enable us to compare risk aversion among individuals as required by these theorems. ${ }^{2}$

\section{Concluding Remarks}

We started our discussion on the rules of stochastic dominance describing them as the rules designed to identify the elements of the Minimal Agreement Set $S(U)$ defined on the set of available lottery-pairs, given a set of utility functions $U$. We know that this Minimal Agreement Set may be very small and even may not exist. Whether such a set exists or not depends on the set $U$ and the set of available lotteries $g(x)=\left\{g^{i}(x)\right\}$. For example, if $g(x)$ contains all continuous lotteries defined on $w^{n}$ and $U=U^{1}$, then the Minimal Agreement Set $S(U) \neq \varnothing$. However, if $g(x)$ is a proper subset of the set of continuous lotteries, the Minimal Agreement Set may be empty. For example, let $g(x)$ be a set of symmetric, two-parameter, continuous probability density functions (lotteries) with the same mean but differing variance. ${ }^{3}$ With $U=U^{1}, S(U)$ does not exist, because the ranking made by the risk-averse individuals will be exactly opposite to that of the risk-loving individuals. On the other hand with $U=U^{2}$ (concave utility functions) the ranking of any two lotteries will be same across all the individuals. It is, therefore, interesting to examine the class of possible restrictions on $g(x)$ and $U$ which guarantee the existence of the Minimal Agreement Set. Since $g(x)$ and $U$

Originally published in Essays in Economic Analysis and Policy

(ed. D. Banerjee), Oxford University Press, 113-30. 


\section{BISWAS Weak*Axiom of Independence}

are sets of functions, the existence theorems are likely to be quite complicated and are certainly beyond the scope of the present paper.

\section{Footnotes}

${ }^{1} S^{1}$ is the graph (without the diagonal elements) of the pre-order induced by $u^{1}$ on $g(x)$. Note, the diagonal of the graph is eliminated by assuming that $\Pi$ does not include pairs of identical lotteries. For the definition of graphs of a preordering and the diagonal see Nachbin (1965).

${ }^{2}$ In the multivariate case, the implication of FSDU is as follows. Consider a group of individuals having different positive monotonic VNM utility functions but identical underlying preference orderings (indifference curves). If $g^{1}(x)$ has FSDU over $g^{2}(x)$ for an individual within the group then $g^{1}(x)$ is preferred to $g^{2}(x)$ by everyone in the group.

${ }^{3}$ For example, consider the class of uniform distribution functions defined within the range [a,b] with mean $(b+a) / 2$.

\section{References}

Arrow, K.J. (1971), Essays in the Theory of Risk Bearing, Chicago: Markham.

Biswas, T. (1983), A note on the Generalised Measures of Risk Aversion, Journal of Economic Theory, 29, 347-52

Bradley, M.G. and D.E. Lehman (1985), Instrument Effects and Stochastic Dominance, paper presented at the AEA conference, December.

Fishburn, P.C. (1974), Convex Stochastic Dominance with Continuous Distribution Functions, Journal of Economic Theory, 7, pp. 143-58.

Hadar, J. and W.R. Russell (1969), Rules for Ordering Uncertain Prospects, American Economic Review, 59, 25-34.

Hanoch, G. and H. Levy (1969), Efficiency Analysis of Choices Involving Risk, Review of Economic Studies, 38, 335-46

Karni, E. (1985), Decision Making Under Uncertainty, Cambridge, Massachusetts: Harvard University Press.

Khilstrom, R.E. and L.J. Mirman (1974), Risk Aversion with Many Commodities, Journal of Economic Theory, 8, 361-88

Levhari, D., J. Paroush and B. Peleg 1(975), Efficiency Analysis of Multivariate Distributions', Review of Economic Studies, 42, 87-91.

Machina, M.J. (1982) A Stronger Characterization of Declining Risk Aversion, Econometrica, 50, 1069-79.

Originally published in Essays in Economic Analysis and Policy

(ed. D. Banerjee), Oxford University Press, 113-30. 
Meyer, J. (1977), Second Degree Stochastic Dominance with Respect to a Function, International Economic Review, 18, 477-87.

Nachbin, I. 1965. Topology and Order, Princeton, New Jersey: D. Van Nostrand Company, Inc.

Nielsen, L.T. (1986), Comparative Risk Aversion, Department of Economics, University of Texas at Austin, unpublished manuscript.

Pratt, J.W. (1964), Risk Aversion in the Small and in the Large, Econometrica, 32, 122-36.

Quirk, J.P. and R. Saposnik. (1962), Admissibility and Measurable Utility Functions, Review of Economic Studies, 29, 141-6

Russell, W.R. and T.K. Seo (1978), Ordering Uncertain Prospects: The Multivariate Utility Function Case, Review of Economic Studies, 45, 605-10.

Whitmore, G.A. (1970), Third-degree Stochastic Dominance, American Economic Review, 60, 457-9

Yaari, M.E. (1969), Some Remarks on Measures of Risk Aversion and on their Uses, Journal of Economic Theory, 59, 315-29.

Originally published in Essays in Economic Analysis and Policy

(ed. D. Banerjee), Oxford University Press, 113-30. 